\title{
\begin{tabular}{l|l} 
Mibraries & DSpace@MIT
\end{tabular}
}

\author{
MIT Open Access Articles
}

\section{INTERSTELLAR SCINTILLATION AND THE RADIO COUNTERPART OF THE FAST RADIO BURST FRB 150418}

The MIT Faculty has made this article openly available. Please share how this access benefits you. Your story matters.

Citation: Akiyama, Kazunori, and Michael D. Johnson. “INTERSTELLAR SCINTILLATION AND THE RADIO COUNTERPART OF THE FAST RADIO BURST FRB 150418." The Astrophysical Journal 824.1 (2016): L3. (C) 2016 The American Astronomical Society

As Published: http://dx.doi.org/10.3847/2041-8205/824/1//3

Publisher: IOP Publishing

Persistent URL: http://hdl.handle.net/1721.1/106128

Version: Final published version: final published article, as it appeared in a journal, conference proceedings, or other formally published context

Terms of Use: Article is made available in accordance with the publisher's policy and may be subject to US copyright law. Please refer to the publisher's site for terms of use. 


\title{
INTERSTELLAR SCINTILLATION AND THE RADIO COUNTERPART OF THE FAST RADIO BURST FRB 150418
}

\author{
Kazunori Akiyama ${ }^{1,2,4}$ and Michael D. Johnson ${ }^{3}$ \\ ${ }^{1}$ Massachusetts Institute of Technology, Haystack Observatory, Route 40, Westford, MA 01886, USA; kazu@ haystack.mit.edu \\ ${ }^{2}$ Mizusawa VLBI Observatory, National Astronomical Observatory of Japan, 2-21-1 Osawa, Mitaka, Tokyo 181-8588, Japan \\ ${ }^{3}$ Harvard Smithsonian Center for Astrophysics, 60 Garden Street, Cambridge, MA 02138, USA \\ Received 2016 March 15; revised 2016 May 3; accepted 2016 May 14; published 2016 June 3
}

\begin{abstract}
Keane et al. have recently reported the discovery of a new fast radio burst (FRB), FRB 150418, with a promising radio counterpart at 5.5 and $7.5 \mathrm{GHz}$ - a rapidly decaying source, falling from $200-300 \mu \mathrm{Jy}$ to $100 \mu \mathrm{Jy}$ on timescales of $\sim 6$ days. This transient source may be associated with an elliptical galaxy at redshift $z=0.492$, providing the first firm spectroscopic redshift for an FRB and the ability to estimate the density of baryons in the intergalactic medium via the combination of known redshift and radio dispersion of the FRB. An alternative explanation, first suggested by Williams \& Berger, is that the identified counterpart may instead be a compact active galactic nucleus (AGN). The putative counterpart's variation may then instead be extrinsic, caused by refractive scintillation in the ionized interstellar medium of the Milky Way, which would invalidate the association with FRB 150418. We examine this latter explanation in detail and show that the reported observations are consistent with scintillating radio emission from the core of a radio-loud AGN having a brightness temperature $T_{\mathrm{b}}$ $\gtrsim 10^{9} \mathrm{~K}$. Using numerical simulations of the expected scattering for the line of sight to FRB 150418 , we provide example images and light curves of such an AGN at 5.5 and $7.5 \mathrm{GHz}$. These results can be compared with continued radio monitoring to conclusively determine the importance of scintillation for the observed radio variability, and they show that scintillation is a critical consideration for continued searches for FRB counterparts at radio wavelengths.
\end{abstract}

Key words: galaxies: individual (WISE J071634.59-190039.2) - galaxies: jets - Galaxy: nucleus radio continuum: galaxies - radio continuum: ISM - scattering

\section{INTRODUCTION}

Fast radio bursts (FRBs) are highly dispersed radio transients emitting a radio-flux density of $\sim 1 \mathrm{Jy}$ for only a few milliseconds or less. Since the first discovery reported in 2007 (Lorimer et al. 2007), more than $17 \mathrm{FRBs}^{5}$ (Petroff et al. 2016) have been discovered with the Parkes Radio telescope (e.g., Lorimer et al. 2007; Keane et al. 2012, 2016), the Arecibo observatory (Spitler et al. 2014, 2016), and the Green Bank telescope (Masui et al. 2015). This population of FRBs is highly inhomogeneous, with some showing high circular polarization (Petroff et al. 2015), others showing high linear polarization (Masui et al. 2015), and others with little polarization (Keane et al. 2016). Although most detected FRBs are isolated events, one source has recently been found to be repeating (Spitler et al. 2016).

The origin of the FRBs remains uncertain. Their large dispersion measures, $\sim 400-1600 \mathrm{~cm}^{-3} \mathrm{pc}$, exceed expected values for propagation in the interstellar medium (ISM), suggesting that FRBs are extragalactic at redshifts of $z \sim$ $0.5-1$. An extragalactic pulsed signal enables a direct probe of properties of the intergalactic medium (IGM) through the cold plasma dispersion relationship, just as pulsars are used to probe the ionized ISM of the Milky Way. The short durations and extreme brightness temperatures of FRBs suggest compact progenitors, such as the magnetars (e.g., Lyubarsky 2014), neutron stars (e.g., Totani 2013; Falcke \& Rezzolla 2014; Zhang 2014), white dwarfs (e.g., Kashiyama et al. 2013), and black holes (e.g., Keane et al. 2012), although the repeating

\footnotetext{
4 JSPS Postdoctoral Fellow for Research Abroad

5 http://www.astronomy.swin.edu.au/pulsar/frbcat/
}

FRB is incompatible with the many proposed cataclysmic events.

Recently, Keane et al. (2016, hereafter K16) reported the discovery of a new FRB (FRB 150418) followed by a slower radio transient detected with the Australia Telescope Compact Array (ATCA). The radio light curves of this transient (hereafter J0716-1900) at 5.5 and $7.5 \mathrm{GHz}$ show rapid decay on timescales of $\sim 6$ days. Optical observations with Subaru found that J0716-1900 is associated with the elliptical galaxy WISE J071634.59-190039.2 at the redshift of $0.492 \pm 0.008$. If this galaxy is indeed associated with FRB 150418, then its identification provides the first measured redshift of an FRB. The dispersion measure of the FRB then provides an estimate of the density of the IGM, in this case giving a value consistent with the standard $\Lambda \mathrm{CDM}$ cosmology.

However, scintillation in the ionized ISM can also introduce rapid variability of compact radio sources such as active galactic nuclei (AGNs), pulsars, and masers (see, e.g., Rickett et al. 1984; Rickett 1990; Narayan 1992; Lovell et al. 2008). Indeed, follow-up observations of J0716-1900 by Williams \& Berger (2016b) detected rapidly varying flux density, with some measurements as high as the original detections by K16 following the FRB. Moreover, because the line of sight to FRB 150418 lies close to the Galactic plane (Galactic latitude $b \approx-3^{\circ} 2$ ), it has scattering that is significantly stronger than that at larger latitudes. Consequently, as noted by K16, the effects of scintillation are significant for this FRB and other nearby compact sources and must be considered as a potential source of the rapid variation seen in J0716-1900. If the variation is indeed extrinsic, caused by scintillating emission 
from a compact galactic nucleus, then its association with FRB 150418 is likely spurious.

In this Letter, we study the role of interstellar scintillation in the radio variability of J0716-1900. In Section 2, we briefly introduce the theory of the interstellar scattering and give expected scattering properties along the line of sight to FRB 150418. Then, we present theoretical simulations based on these quantities in Section 3. Finally, we discuss the role of continued follow-up observations of FRB 150418 and general considerations for follow-up of future FRBs in Section 4. Throughout the Letter, we use a $\Lambda$ CDM cosmology with $h=0.705, \Omega_{m}=0.228$, and $\Omega_{\Lambda}=0.726$ (Komatsu et al. 2009), providing the luminosity distance and linear scale of $2808 \mathrm{Mpc}$ and $6.1 \mathrm{pc}$ mas $^{-1}$ for J0716-1900, respectively.

\section{SCATTERING AND SCINTILLATION OF J0716-1900}

\subsection{Scattering Theory}

Scattering of radio waves in the interstellar plasma arises from fluctuations in electron density. A variety of measurements find that the three-dimensional power spectrum $P(\boldsymbol{q})$ of the density fluctuations corresponds to a turbulent cascade that is injected on scales of $\gg 1$ au and is dissipated on scales of $\sim 10^{2} \mathrm{~km}: P(\boldsymbol{q}) \propto|\boldsymbol{q}|^{-(\alpha+2)}$, with $\alpha$ close to the value of $5 / 3$ expected for Kolmogorov turbulence (Armstrong et al. 1995). In many instances, the scattering properties can be well described by a single, thin phase-changing screen $\varphi(\boldsymbol{x})$, where $\boldsymbol{x}$ is a transverse coordinate on the screen. The statistical characteristics of the scattering and scintillation can then be related to statistical characteristics of the phase fluctuations through a spatial structure function $D_{\varphi}(\boldsymbol{x})=\left\langle\left[\varphi\left(\boldsymbol{x}+\boldsymbol{x}_{0}\right)-\varphi\left(\boldsymbol{x}_{0}\right)\right]^{2}\right\rangle_{x_{0}}$. Density fluctuations that follow the above power law then give rise to a power-law structure function, $D_{\varphi}(\boldsymbol{x}) \propto|\boldsymbol{x}|^{\alpha}$.

The properties of the scattering screen are characterized by a pair of length scales. The phase coherence length (or diffractive scale), $r_{0} \propto \lambda^{-2 / \alpha}$, decreases with increasing observing wavelength $\lambda$ and determines the scale at which the screen phase decorrelates: $D_{\varphi}\left(r_{0}\right) \equiv 1$. The Fresnel scale, $r_{\mathrm{F}} \equiv \sqrt{\frac{\lambda D}{2 \pi}}$, depends on the distance $D$ from the observer to the scattering screen and determines how the geometrical phase of propagation varies across the screen. For radio observations, interstellar scattering is usually in the strong scattering regime, corresponding to the condition $r_{0} \ll r_{\mathrm{F}}$ (i.e., $D_{\varphi}\left(r_{\mathrm{F}}\right) \gg 1$ ), and a third scale becomes important: the refractive scale, $r_{\mathrm{R}}=r_{\mathrm{F}}^{2} / r_{0} \propto \lambda^{1+2 / \alpha}$, which determines the size of the scattered image of the point source.

Scintillation in strong scattering is dominated by two distinct branches, diffractive and refractive, on these widely separated scales. Diffractive scintillation, arising from fluctuations on scales of $r_{0}$, is quenched by a source exceeding the angular scale $r_{0} / D$. As a result, diffractive scintillation is typically quenched by AGNs. Refractive scintillation arises from fluctuations on scales of $r_{\mathrm{R}}$ and is only quenched by a source exceeding the angular scale $r_{\mathrm{R}} / D$ so can persist for compact AGNs. With the characteristic transverse velocity of the scattering material $v_{\perp}$, the diffractive and refractive timescales are given by $t_{0} \equiv r_{0} / v_{\perp}$ and $t_{\mathrm{R}} \equiv r_{\mathrm{R}} / v_{\perp}$. For pulsars, $v_{\perp} \sim$ $10^{7} \mathrm{~cm} \mathrm{~s}^{-1}$ is typical (e.g., Cordes et al. 1988; Rickett 1990), but this is typically dominated by proper motion of the pulsar. For an extragalactic source, the velocity is determined by a combination of motion of the Earth and of the scattering material, and we adopt a characteristic velocity of $v_{\perp}=5 \times 10^{6} \mathrm{~cm} \mathrm{~s}^{-1}$ (see, e.g., Rickett et al. 1995).

The flux variability due to the scattering effects is often quantified with the modulation index, defined by $m \equiv \sqrt{\left\langle F_{\nu}^{2}\right\rangle-\left\langle F_{\nu}\right\rangle^{2}} /\left\langle F_{\nu}\right\rangle$, where $F_{\nu}$ is the flux density. For refractive scintillation, the modulation index for a source with angular size $\theta_{\text {src }}$ smaller than $\theta_{\text {scatt }}$ (i.e., the refractive scale) is $m \approx\left(r_{0} / r_{\mathrm{F}}\right)^{2-\alpha}$, where the precise prefactor is of order unity (e.g., Goodman \& Narayan 1985; Narayan 1992). Larger sources suppress the modulation index by a factor of $\left(\theta_{\text {scatt }} / \theta_{\text {src }}\right)^{2-\alpha / 2}$, where $\theta_{\text {src }}$ is the unscattered source size.

\subsection{Expected Scattering Properties for J0716-1900}

We can estimate characteristic scattering properties for J0716-1900 with the Galactic free electron density model, NE2001 ${ }^{6}$ (Cordes \& Lazio 2002, 2003). For this model, the expected FWMH scattering size along the line of sight to $\mathrm{J} 0716-1900$ at $1 \mathrm{GHz}$ is $\theta_{\text {scatt }, 1 \mathrm{GHz}}=4.5$ mas. Due to its low Galactic latitude, this value is significantly higher than the median angular broadening at $1 \mathrm{GHz}$ ( $\sim 1$ mas). Leaving $\theta_{\text {scatt } 1 \mathrm{GHz}}$ as a free parameter to provide formulas that are applicable to arbitrary lines of sight (but referencing to our assumed value for J0716-1900), other scattering parameters can then be estimated as follows:

$$
\begin{aligned}
& \theta_{\text {scatt }}=4.5 \mathrm{mas} \times\left(\frac{\theta_{\text {scatt }, 1 \mathrm{GHz}}}{4.5 \mathrm{mas}}\right)\left(\frac{\nu}{1 \mathrm{GHz}}\right)^{-1-\frac{2}{\alpha}} \\
& r_{0}=2.1 \times 10^{8} \mathrm{~cm} \times\left(\frac{\theta_{\text {scatt }, 1 \mathrm{GHz}}}{4.5 \mathrm{mas}}\right)^{-1}\left(\frac{\nu}{1 \mathrm{GHz}}\right)^{\frac{2}{\alpha}} \\
& r_{\mathrm{F}}=1.2 \times 10^{11} \mathrm{~cm} \times\left(\frac{\nu}{1 \mathrm{GHz}}\right)^{-\frac{1}{2}}\left(\frac{D}{1 \mathrm{kpc}}\right)^{\frac{1}{2}} \\
& r_{\mathrm{R}}=6.8 \times 10^{13} \mathrm{~cm} \times\left(\frac{\theta_{\text {scatt }, 1 \mathrm{GHz}}}{4.5 \mathrm{mas}}\right)\left(\frac{\nu}{1 \mathrm{GHz}}\right)^{-1-\frac{2}{\alpha}} \\
& \times\left(\frac{D}{1 \mathrm{kpc}}\right) \\
& t_{0}=44 \mathrm{~s} \times\left(\frac{\theta_{\text {scatt, } 1 \mathrm{GHz}}}{4.5 \mathrm{mas}}\right)^{-1}\left(\frac{\nu}{1 \mathrm{GHz}}\right)^{\frac{2}{\alpha}}\left(\frac{v_{\perp}}{50 \mathrm{~km} \mathrm{~s}^{-1}}\right)^{-1} \\
& t_{\mathrm{R}}=160 \text { days } \times\left(\frac{\theta_{\text {scatt }, 1 \mathrm{GHz}}}{4.5 \mathrm{mas}}\right)\left(\frac{\nu}{1 \mathrm{GHz}}\right)^{-1-\frac{2}{\alpha}}\left(\frac{D}{1 \mathrm{kpc}}\right) \\
& \times\left(\frac{v_{\perp}}{50 \mathrm{~km} \mathrm{~s}^{-1}}\right)^{-1} \\
& m \approx 12 \% \times\left(\frac{\theta_{\text {scatt }, 1 \mathrm{GHz}}}{4.5 \mathrm{mas}}\right)^{-(2-\alpha)}\left(\frac{\nu}{1 \mathrm{GHz}}\right)^{\frac{4}{\alpha}-\frac{\alpha}{2}-1} \\
& \times\left(\frac{D}{1 \mathrm{kpc}}\right)^{-\frac{1}{6}} \text {. }
\end{aligned}
$$

In Table 1, we give characteristic quantities for a screen at $D=1 \mathrm{kpc}$ at $1,5.5$, and $7.5 \mathrm{GHz}$ with a power-law index of $\alpha=5 / 3$. We emphasize that these results are only appropriate in the strong scattering regime, so for J0716-1900 they are applicable at frequencies below $\sim 40 \mathrm{GHz}$. This weak/strong

\footnotetext{
http://www.nrl.navy.mil/rsd/RORF/ne2001/
} 
Table 1

Estimated Scattering Properties for J0716-1900

\begin{tabular}{llccc}
\hline \hline Quantity & Unit & $1 \mathrm{GHz}$ & $5.5 \mathrm{GHz}$ & $7.5 \mathrm{GHz}$ \\
\hline$\theta_{\text {scatt }}$ & $(\mathrm{mas})$ & 4.5 & 0.11 & 0.054 \\
$r_{0}$ & $(\mathrm{~cm})$ & $2.2 \times 10^{8}$ & $1.7 \times 10^{9}$ & $2.4 \times 10^{9}$ \\
$r_{\mathrm{F}}$ & $(\mathrm{cm})$ & $1.2 \times 10^{11}$ & $5.2 \times 10^{10}$ & $4.4 \times 10^{10}$ \\
$r_{\mathrm{R}}$ & $(\mathrm{cm})$ & $6.8 \times 10^{13}$ & $1.6 \times 10^{12}$ & $8.0 \times 10^{11}$ \\
$t_{0}$ & $(\mathrm{~s})$ & 44 & 340 & 490 \\
$t_{\mathrm{R}}$ & $(\mathrm{d})$ & 160 & 3.7 & 1.9 \\
$m$ & $(\%)$ & 12 & 32 & 38 \\
\hline
\end{tabular}

transition frequency is much higher than that of most lines of sight because of the low Galactic latitude of J0716-1900.

The most important implication from Table 1 is that J07161900 can be highly affected by refractive scattering at 5.5 and $7.5 \mathrm{GHz}$ if the source is more compact than $\sim 0.1$ mas (corresponding to a distance of $\sim 0.6 \mathrm{pc}$ at the redshift of $z=0.492)$. This upper limit is reasonable for the radio core of a relativistic jet (e.g., as has been seen directly with space very long baseline interferometry, VLBI; Horiuchi et al. 2004). We also note that recent VLBI surveys have shown that fainter sources are more likely to be dominated by compact components (e.g., Deller \& Middelberg 2014), and preliminary results of VLBI observations show that J0716-1900 is unresolved on milliarcsecond scales (Bassa et al. 2016; Marcote et al. 2016). Refractive scattering would then cause modulation of $\sim 30 \%$ on a timescale of a few days, similar to what has been observed (see Figure 2). Williams \& Berger (2016b) have also obtained a similar conclusion.

Thus, in addition to the importance of scintillation for the radio variability a compact afterglow, as noted by $\mathrm{K} 16$, refractive scintillation is also a critical consideration even for compact emission from an AGN, and the radio variability of J0716-1900 is comparable to the expected refractive scintillation in the ISM. Note also that the pulse broadening due to the interstellar scintillation is only $\sim 0.02 \mathrm{~ms}$ at $1 \mathrm{GHz}$, which is much shorter than the observed pulse duration of $0.8 \pm 0.3 \mathrm{~ms}$ for FRB 150418 (K16).

\section{NUMERICAL SIMULATIONS}

To further study the radio-flux variation caused by the interstellar scintillation and understand how the variations may be correlated at 5.5 and $7.5 \mathrm{GHz}$, we performed numerical simulations of the scattering of J0716-1900. Following Johnson \& Gwinn (2015), we generated scattered images at 5.5 and $7.5 \mathrm{GHz}$ for an intrinsic source that was a circular Gaussian with an FWHM size of 0.1 mas. ${ }^{7}$ We generated a scattering screen with $2^{14} \times 2^{14}$ random phases with the characteristic scattering parameters given in Table 1. Our simulations span 1207 days $\left(\sim 300-600 t_{\mathrm{R}}\right)$ with a time resolution of 0.27 day $\left(\ll t_{\mathrm{R}}\right)$. To investigate the variability statistics, we also generated $\sim 200$ different scattering realizations, providing a total span of $\sim 2 \times 10^{5}$ days. We show example images of the scattered structure for one realization of the scattering in Figure 1. We calculated the total flux density of each scattered image at each time to generate a light curve for each frequency.

In Figure 2, we show simulated light curves at 5.5 and 7.5 GHz, and in Figure 3, we show the probability distribution

\footnotetext{
7 Because the scattering has deterministic frequency dependence, a single scattering screen determines the scattered image at all frequencies.
}

for the simulated light curves. The light curves exhibit the expected fast variability discussed in Section 2.2. The modulation index is $\sim 29 \%$ and $\sim 25 \%$ for the whole data at 5.5 and $7.5 \mathrm{GHz}$, respectively, consistent with the original observations of the ATCA (K16) and also with the follow-up observations with the Very Large Array (VLA; Vedantham et al. 2016; Williams \& Berger 2016a, 2016b). For direct comparison with these observations, we also compare light curves for 400 days with the normalized light curve of K16, Williams \& Berger (2016a, 2016b), Vedantham et al. (2016), Bassa et al. (2016), and Marcote et al. (2016) in Figure 2(b). The simulated light curves vary on scales of $\sim 3-5$ days, consistent with all observations, and have highly correlated variability at 5.5 and $7.5 \mathrm{GHz}$, again consistent with the synchronized flux variation reported by K16. Refractive scintillation can also explain the gentle spectral modulation across 2-18 GHz reported by Vedantham et al. (2016).

\section{THE ORIGIN OF J0716-1900}

We have shown that refractive scattering can potentially explain the amplitude and timescale of the radio variations of J0716-1900. This explanation requires that the source is sufficiently compact, most plausibly a weak, radio-loud AGN. This explanation is consistent with preliminary results of VLBI observations (Bassa et al. 2016; Marcote et al. 2016). Here, we briefly discuss expected observational properties for this scenario.

The typical flux density of $\sim 0.1-0.3 \mathrm{mJy}$ of J0716-1900 (K16) corresponds to the radio power of

$$
P_{\nu}=9.4 \times 10^{22} \mathrm{~W} \mathrm{~Hz}^{-1} \times\left(\frac{F_{\nu}}{0.1 \mathrm{mJy}}\right)\left(\frac{D_{\mathrm{L}}}{2808 \mathrm{Mpc}}\right)^{2} .
$$

This power is consistent with the typical nuclear radio power of the nearby low-luminosity AGNs (LLAGNs) and elliptical galaxies (e.g., Doi et al. 2011) that are thought to be powered by a hot accretion flow (e.g., Yuan \& Narayan 2014) or a faint jet (e.g., Falcke et al. 2004). This radio power is also consistent with the low-power end of the known blazars (Liuzzo et al. 2013; Massaro et al. 2015).

To avoid quenching the refractive scintillation, the source angular size must subtend $\theta_{\text {src }} \lesssim 0.1$ mas, providing a lower limit on its brightness temperature:

$$
\begin{aligned}
T_{\mathrm{b}} & =\frac{c^{2}}{2 k_{\mathrm{B}} \nu^{2}} \frac{F_{\nu}}{\pi\left(\theta_{\mathrm{src}} / 2\right)^{2} \ln 2} \\
& \gtrsim 8.4 \times 10^{8} \mathrm{~K} \times\left(\frac{\nu}{5.5 \mathrm{GHz}}\right)^{-2}\left(\frac{F_{\nu}}{0.1 \mathrm{mJy}}\right)\left(\frac{\theta_{\mathrm{src}}}{0.1 \mathrm{mas}}\right)^{2} .
\end{aligned}
$$

The lower limit of the brightness temperature is compatible with low-power radio galaxies and blazars (e.g., Liuzzo et al. 2009, 2013; Piner \& Edwards 2014). Thus, in addition to the source size, the radio power and the brightness temperature are also reasonable as nuclear emission from an LLAGN, low-powered blazar, or weak AGN.

We note that Vedantham et al. (2016) have very recently measured a flat radio spectrum for J0716-1900, which is generally seen in blazars (e.g., Massaro et al. 2014). The flat radio spectrum suggests that the majority of the arcsecond-scale flux density originates in the optically thick radio core emission, which could be sufficiently compact to be 


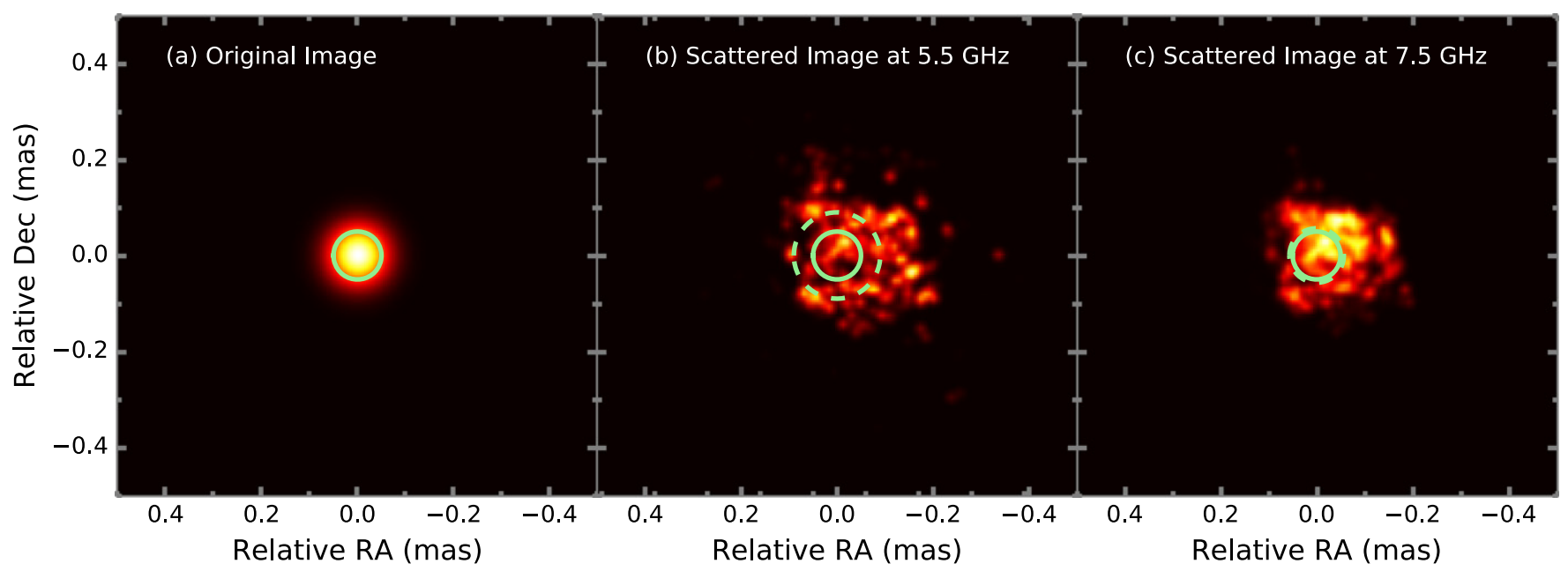

Figure 1. Simulated images showing the effects of refractive substructure at $\nu=5.5$ and $7.5 \mathrm{GHz}$. Panel (a) shows the circular Gaussian intrinsic source with the FWHM size of 0.1 mas, which is shown in a circle with the solid line. Panels (b) and (c) show snapshot images of the scattered structure at 5.5 and $7.5 \mathrm{GHz}$. The scattering parameters correspond to the NE2001 estimates for J0716-1900 (see Section 2.2). The dashed lines indicate the ensemble-average scattered size, $\sqrt{\theta_{\mathrm{scatt}}^{2}+\theta_{\mathrm{src}}^{2}}$. Other effects from refractive scattering are also apparent, such as shifts in the image centroids.
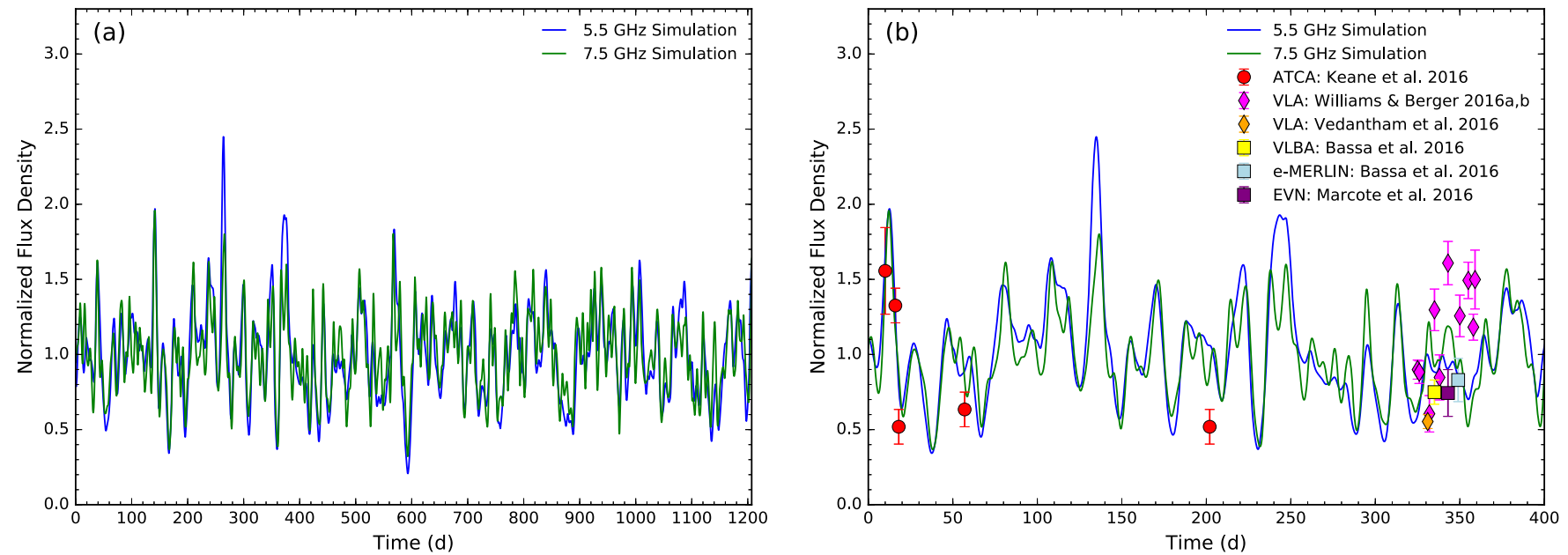

Figure 2. Simulated light curves at $5.5 \mathrm{GHz}$ (blue line) and $7.5 \mathrm{GHz}$ (green line) for 1207 days (a) and for 400 days following a flare-like scintillation feature (b). Each light curve is normalized by its mean value. As expected, the fluctuations at these two frequencies are tightly correlated. For reference, the $5.5 \mathrm{GHz}$ data of $\mathrm{K} 16$ with ATCA, Williams \& Berger (2016a, 2016b) and Vedantham et al. (2016) with VLA, Bassa et al. (2016) with VLBA and e-MERLIN, and Marcote et al. (2016) with EVN are also shown after being normalized by the mean value of all data.
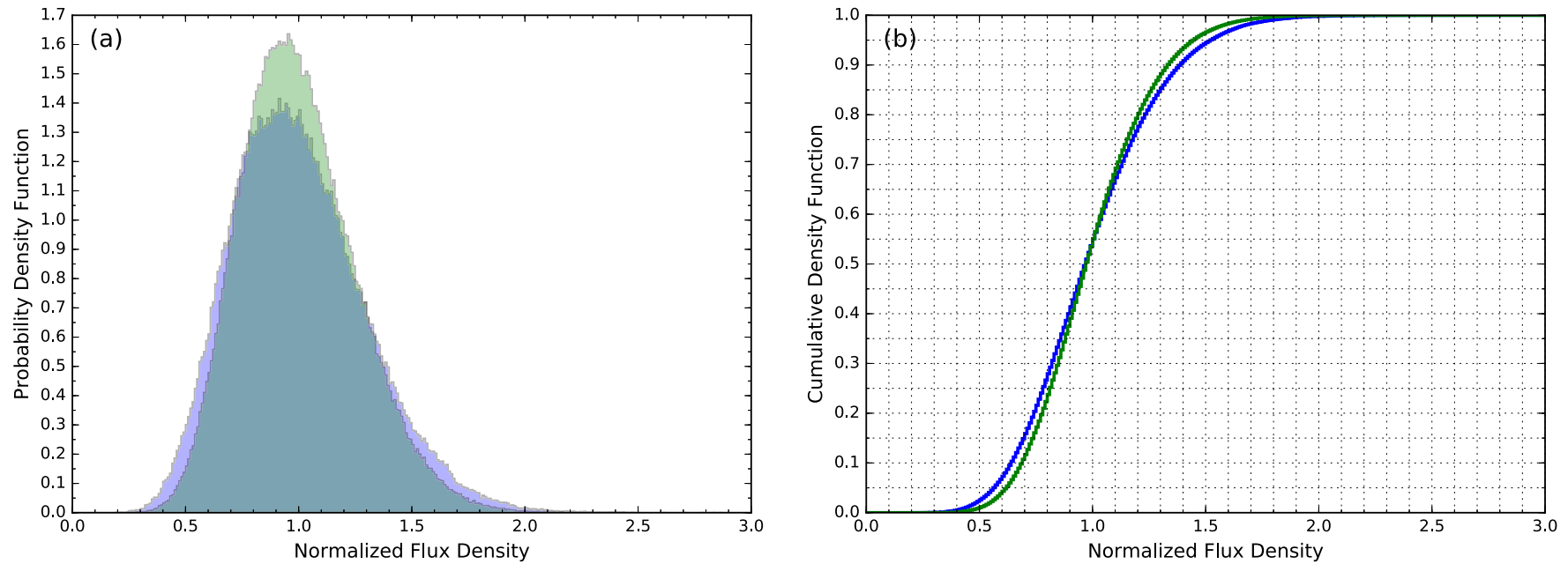

Figure 3. Probability distributions of the radio flux at $5.5 \mathrm{GHz}$ (blue line) and $7.5 \mathrm{GHz}$ (green line) obtained from all simulated data (a total of $\sim 2 \times 10^{5}$ days) and normalized by their mean values. Panel (a) shows the probability density function (PDF); panel (b) shows the cumulative distribution function (CDF). For a timeindependent intrinsic source, the PDF of a complete scattering ensemble is expected to follow a Rice distribution. 
scintillating, and other observations have revealed the presence of compact jet structure in elliptical galaxies without any feature of AGNs in the optical/infrared spectrum (e.g., Akiyama et al. 2016). Thus, the radio spectrum supports the scenario in which the variability J0716-1900 is predominantly from interstellar scintillation.

Williams \& Berger (2016b) were the first to argue that the radio emission from J0716-1900 arises from an AGN and have noted that the observed variability is incompatible with standard afterglow evolution. Based on the deep VLA imaging study of Fomalont et al. (1991) at $5 \mathrm{GHz}$, Williams \& Berger (2016b) further note that $\sim 16$ sources above $100 \mu \mathrm{Jy}$ are expected per Parkes beam. Moreover, Fomalont et al. (1991) found that most sources between 60 and $1000 \mu \mathrm{Jy}$ were unresolved $(<1$ ".5). These estimates are then favorable for ascribing the variability of J0716-1900 to refractive scintillation. However, we caution that the variability of J0716-1900 should not be directly compared with variability reported in other surveys at these frequencies because its line of sight is close to the Galactic plane and so has significantly stronger scattering than the median Galactic values. Consequently, the transition to weak scattering, where the refractive modulation index $m$ peaks, is at higher frequencies for J0716-1900 than for higher-latitude sources.

\section{SUMMARY}

In short, the fast variation of J0716-1900 can be reasonably explained as refractive scintillation in the ISM and may not represent an afterglow associated with FRB 150418. Both the analytical theory of refractive scattering and our numerical simulations show that the expected scattering of J0716-1900 can reproduce the observed timescales and modulation index at 5.5 and $7.5 \mathrm{GHz}$. They also naturally explain the synchronized modulation at these frequencies and the gentle modulation across the wider radio spectrum reported by Vedantham et al. (2016). Refractive scintillation requires that the source size is smaller than $\lesssim 0.1$ mas $(\lesssim 0.6 \mathrm{pc}$ at the source), which is consistent with preliminary results of VLBI observations (Bassa et al. 2016; Marcote et al. 2016). This source size corresponds to a brightness temperature $T_{\mathrm{b}}>10^{9} \mathrm{~K}$, compatible with LLAGNs and faint blazars. Our results would also apply to more compact emission, such as an FRB afterglow, and demonstrate that fast variability does not necessitate a high Doppler factor.

Our estimates of the scattering are not sensitive to assumptions about the location of the scattering screen, but our derived timescales are uncertain by a factor of several, both from the unknown velocity of the scattering material and the unknown distance of the scattering from the Earth. Also, our estimates of the scattering of J0716-1900 (from the NE2001 model) are uncertain by a factor of several. Finally, refractive scintillation can only cause the observed flux variability if the majority of the source flux originates in the compact core emission. The core dominance of J0716-1900 is therefore one of the key questions for continued studies and can be confirmed using VLBI. Even with these cautions and remaining uncertainties, it is evident that refractive scintillation is of fundamental importance for the interpretation of J0716-1900 and for radio identification of FRB afterglows more generally.

K.A. is financially supported by JSPS Postdoctoral Fellowships for Research Abroad and grants from the National Science Foundation (NSF). M.D.J. thanks the Gordon and Betty Moore Foundation for financial support (\#GBMF-3561). We also thank Dr. Hiroshi Nagai for helpful discussions on FRB 150418. We thank Dr. Katherine Rosenfeld for writing ScatterBrane, the software used for our scattering simulations (http://krosenfeld.github.io/scatterbrane).

\section{REFERENCES}

Akiyama, K., Stawarz, Ł., Tanaka, Y. T., et al. 2016, ApJL, 823, L26 Armstrong, J. W., Rickett, B. J., \& Spangler, S. R. 1995, ApJ, 443, 209 Bassa, C., Beswick, R., Tingay, S. J., et al. 2016, ATel, 8938 Cordes, J. M., \& Lazio, T. J. W. 2002, arXiv:astro-ph/0207156 Cordes, J. M., \& Lazio, T. J. W. 2003, arXiv:astro-ph/0301598 Cordes, J. M., Rickett, B. J., \& Backer, D. C. (ed.) 1988, AIP Conf. Ser. 174, Radio Wave Scattering in the Interstellar Medium; Proc. AIP Conf. (San Diego, CA: Univ. California)

Deller, A. T., \& Middelberg, E. 2014, AJ, 147, 14

Doi, A., Nakanishi, K., Nagai, H., Kohno, K., \& Kameno, S. 2011, AJ, 142,167

Falcke, H., Körding, E., \& Markoff, S. 2004, A\&A, 414, 895

Falcke, H., \& Rezzolla, L. 2014, A\&A, 562, A137

Fomalont, E. B., Windhorst, R. A., Kristian, J. A., \& Kellerman, K. I. 1991, AJ, 102,1258

Goodman, J., \& Narayan, R. 1985, MNRAS, 214, 519

Horiuchi, S., Fomalont, E. B., Taylor, W. K., et al. 2004, ApJ, 616, 110

Johnson, M. D., \& Gwinn, C. R. 2015, ApJ, 805, 180

Kashiyama, K., Ioka, K., \& Mészáros, P. 2013, ApJL, 776, L39

Keane, E. F., Johnston, S., Bhandari, S., et al. 2016, Natur, 530, 453

Keane, E. F., Stappers, B. W., Kramer, M., \& Lyne, A. G. 2012, MNRAS, 425, L71

Komatsu, E., Dunkley, J., Nolta, M. R., et al. 2009, ApJS, 180, 330

Liuzzo, E., Giovannini, G., Giroletti, M., \& Taylor, G. B. 2009, A\&A, 505,509

Liuzzo, E., Giroletti, M., Giovannini, G., et al. 2013, A\&A, 560, A23

Lorimer, D. R., Bailes, M., McLaughlin, M. A., Narkevic, D. J., \& Crawford, F. 2007, Sci, 318, 777

Lovell, J. E. J., Rickett, B. J., Macquart, J.-P., et al. 2008, ApJ, 689, 108

Lyubarsky, Y. 2014, MNRAS, 442, L9

Marcote, B., Giroletti, M., Garrett, M., et al. 2016, ATel, 8959

Massaro, E., Maselli, A., Leto, C., et al. 2015, Ap\&SS, 357, 75

Massaro, F., Giroletti, M., D’Abrusco, R., et al. 2014, ApJS, 213, 3

Masui, K., Lin, H.-H., Sievers, J., et al. 2015, Natur, 528, 523

Narayan, R. 1992, RSPTA, 341, 151

Petroff, E., Bailes, M., Barr, E. D., et al. 2015, MNRAS, 447, 246

Petroff, E., Barr, E. D., Jameson, A., et al. 2016, PASA, submitted (arXiv:1601.03547)

Piner, B. G., \& Edwards, P. G. 2014, ApJ, 797, 25

Rickett, B. J. 1990, ARA\&A, 28, 561

Rickett, B. J., Coles, W. A., \& Bourgois, G. 1984, A\&A, 134, 390

Rickett, B. J., Quirrenbach, A., Wegner, R., Krichbaum, T. P., \& Witzel, A. 1995, A\&A, 293, 495

Spitler, L. G., Cordes, J. M., Hessels, J. W. T., et al. 2014, ApJ, 790, 101

Spitler, L. G., Scholz, P., Hessels, J. W. T., et al. 2016, Natur, 531, 202

Totani, T. 2013, PASJ, 65, L12

Vedantham, H. K., Ravi, V., Mooley, K., et al. 2016, ApJL, submitted (arXiv:1603.04421)

Williams, P. K. G., \& Berger, E. 2016a, ATel, 8946

Williams, P. K. G., \& Berger, E. 2016b, ApJL, 821, L22

Yuan, F., \& Narayan, R. 2014, ARA\&A, 52, 529

Zhang, B. 2014, ApJL, 780, L21 